\title{
A novel concept on volumetric assessment and quantification of gastrointestinal bleed on computed tomography angiography: Observations based on a case series
}

\author{
Amritha P. P., Karthik Gadabanahalli, Venkatraman Bhat, and Kishore Kumar B. N. \\ Department of Radiology, Mazumdar Shaw Medical Centre - Narayana Multispecialty Hospital, \\ Narayana Health, Bommasandra, Bengaluru, India
}

\begin{abstract}
Estimation of quantity and rate of bleeding is of great value in the management of patients with acute GI bleed. Endoscopy and multiphase contrast enhanced computed tomography (MCECT) are the presently employed two important methods for this purpose. Still there is a lacuna in the methods of precise estimation of amount of bleed, which at the moment are somewhat unreliable and subjectively evaluated. We present the value of dynamic contrast-enhanced CT examination in quantitatively estimating the amount of extravasated blood with the help of three illustrative clinical examples. Technique CT examination, the methodology for assessment of quantity of bleed is presented and the discussion of existing literature regarding the estimation methods is presented. (Ann Hepatobiliary Pancreat Surg 2021;25: 160-166)
\end{abstract}

Key Words: GI bleed; Quantitative CT; Multiphasic contrast enhanced CT; MCECT; Rate of GI bleed; Volume estimation of $\mathrm{Gl}$ bleed

\section{INTRODUCTION}

Acute gastrointestinal bleeding is an acute medical condition which warrants rapid diagnosis and management. It is one of the leading causes of high morbidity and mortality among patients presenting to the emergency department. ${ }^{1}$ Methods of evaluation of suspected gastrointestinal bleed include endoscopy/colonoscopy, radionuclide imaging, CT angiography, CT enterography, catheter angiography, push endoscopy and capsule endoscopy etc. ${ }^{2}$ None of the above methods at the present provide unequivocal information on quantity of GI bleed. The quantification and rate of bleed helps the clinician to assess the imminent clinical status and strategy for management. It helps to stratify patients into those with high or low rates of bleed, thus stratifying patients for urgent management.

\section{ROLE OF MCECT}

CT angiography is an extremely useful and cost-effective modality in the assessment of gastrointestinal bleeding. ${ }^{3}$ Plain CT demonstrates bleed as areas of hyper-density in the lumen. With the use of intravenous contrast agents, the site of bleed can be localized and nature of the bleeder (arterial or venous) can be delineated. This information provides essential data for identification and access to the vessel causing the acute bleeding, thus planning the need for therapeutic embolization or bowel packing.

CT particularly has major advantages over other techniques in patients with severe bleeding. ${ }^{4}$ In some cases endoscopy or colonoscopy may be ineffective as the endoscope may not be passed up to the bleeding site for various reasons. Contexts include situations where bowel preparation is inadequate or in acute cases of bleeding wherein visibility of the bleeding vessel is compromised due to excessive blood products and clots in the bowel lumen. ${ }^{5}$

Received: May 18, 2020; Revised: July 24, 2020; Accepted: July 25, 2020

Corresponding author: Venkatraman Bhat

Department of Radiology, Mazumdar Shaw Medical Centre - Narayana Multispecialty Hospital, Narayana Health, Bommasandra 560099, Bengaluru, India

Tel: +9481027387, E-mail: bvenkatraman@gmail.com

Copyright (C) 2021 by The Korean Association of Hepato-Biliary-Pancreatic Surgery

This is an Open Access article distributed under the terms of the Creative Commons Attribution Non-Commercial License (http://creativecommons.org/ licenses/by-nc/4.0) which permits unrestricted non-commercial use, distribution, and reproduction in any medium, provided the original work is properly cited. Annals of Hepato-Biliary-Pancreatic Surgery • pISSN: 2508-5778 - eISSN: 2508-5859 
CT also serves as a versatile, fast initial modality as the investigation is completed quickly and large active bleeding can be identified easily along with additional anatomical information of the adjacent organs.

\section{MCECT TECHNIQUE}

Three-phase examination consisting of unenhanced, arterial and portal venous phases is performed in CT angiography of abdomen in patients with acute gastrointestinal bleeding. The scan should include the complete abdomen and pelvis, from the diaphragm to the inferior pubic ramus. No oral contrast material is generally administered. Intravenous contrast material is administered through an antecubital vein with a power injector at a rate of 4 $\mathrm{ml} / \mathrm{sec}$, followed by a $50 \mathrm{ml}$ of saline chaser. The dose is adjusted for body weight and iodine concentration, with the total volume of contrast material typically varying between 100 and $125 \mathrm{ml}$ of an agent high in iodine concentration $(>300 \mathrm{mg}$ of iodine per $\mathrm{ml}){ }^{6}$

Our examinations were performed on a 128 slice scanner using helical acquisition of images. A plain CT was performed without oral contrast, followed by dual phase contrast examination with intravenous contrast (1.2-1.5 $\mathrm{ml} / \mathrm{kg}$ ) given at the rate of $4-5 \mathrm{ml} / \mathrm{sec}$. Arterial phase images were obtained using automated bolus tracking with triggering ROI on upper abdominal aorta and delayed phase obtained 45-50 seconds later. Images were obtained at 120 $\mathrm{Kv}$ and 300-330 mAs for adults (100-120 kV and 120-150 $\mathrm{mAs}$ for the child). Slices were acquired at $5 \mathrm{~mm}$ thickness and reconstructed to $0.675 \mathrm{~mm}$ sections. Coronal and Sagittal reformatting were done. Visual inspection of plain and contrast images were done and using Philips Intellispace software version 11.0 (Koninklije Philips NV, Netherlands). The contrast extravasated area with a $\mathrm{HU}$ of more than 90 was seeded manually and its volume was estimated by the software. Based on the time elapsed between the phases, and estimated volume of contrast that has extravasated, the likely rate of bleeding was calculated.

\section{VOLUMETRIC ASSESSMENT OF RATE OF BLEEDING}

We performed $3 \mathrm{D}$ volumetric assessment in three cases of massive bleeding. Follow on endoscopic examination showed significant blood clots, however no bleeder was identified. Quantitative estimation of clot volume was done by endoscopist, subjectively.

On work station volumetric assessment of extravasated contrast was done. DICOM images were opened using "CT viewer" application in Philips Intellispace version 11.0. After visual inspection of plain and contrast images, the contrast extravasated area with an HU of more than +90 was seeded manually using "Clip and 3D segmentation". Its volume was calculated by the software. Using "Tissue management", images were then volume rendered to produce a 3D display and volume was calculated. Generally volume estimation of bleed is made on a formula 'ABC/2x slice thickness' wherein A, B and C represent dimensions of selected attenuation profile. This formula is extensively used in the volume assessment of intracranial bleed. ${ }^{7}$ Rate of bleed is a guesstimate, done and documented with the help two data sets, i.e. arterial and portal venous phase. Arterial values are determined by the point of bolus triggering. Portal phase obtained after 50 seconds. Volume change elapsed between the two phases of CT acquisition when standardised for unit time, allows calculation of rate of bleeding. Estimated volumes were compared. Higher rate of bleed identified patients who are in need to urgent management.

\section{CASE}

\section{Case 1}

3-year-old female, known case of graft-versus-host-disease, following chemotherapy for acute lymphatic leukemia, presented with severe rectal bleed and passage of clots. She underwent colonoscopy which showed erythematous colonic mucosa with multiple erosions. No active bleed was noticed. Her clinical condition worsened with the development of hypotension, hypothermia and was supported with blood products. Her hemoglobin level fell to $10.3 \mathrm{~g} / \mathrm{dl}$ from $10.6 \mathrm{~g} / \mathrm{dl}$ and hematocrit $32.8 \%$ to $30.9 \%$ over 24 hours despite transfusion of blood products. Hence CT was performed. Plain CT showed hyperdense content in transverse, descending and sigmoid colon. Arterial phase imaging did not show any enlarged artery or bleeding vessel. A hyperdense nodular focus was noted in the sigmoid in arterial phase. Progressive increased density of this focus within the sigmoid lumen was noted on delayed im- 
ages suggestive of active contrast extravasation, the volume of which was calculated using software (Fig. 1).

Based on computed estimation, the volume of contrast pooled within lumen between phases, acquired at an interval of 50 seconds, was $15.2 \mathrm{cc}$. Hence rate of bleed was calculated as $0.3 \mathrm{cc} / \mathrm{s}$ or $18 \mathrm{cc} / \mathrm{min}$.

The patient underwent angiography which did not show any active arterial bleeder, hence embolization was not attempted. Emergency sigmoidoscopy examination, showed hematoma in the recto-sigmoid with multiple clots within the lumen. Blood ooze from the base of hematoma was identified. Haemostat application was done after which the bleeding was controlled and patient improved hemodynamically.

\section{Case 2}

38-year-male developed right subdiaphragmatic and subcapsular collection in right lobe of liver two weeks following laparoscopic cholecystectomy for cholelithiasis. Pigtail catheter was placed for drainage of subcapsular collection, following which he developed blood stained discharge from the pigtail catheter. On day 2 of admission he had sudden drop in haemoglobin (from $9.8 \mathrm{~g} / \mathrm{dl}$ to 5.0 $\mathrm{g} / \mathrm{dl}$ and hematocrit fell from $30.6 \%$ to $15.9 \%$ over 48 hours) and desaturation followed by which four units of packed RBC given. UGI endoscopy was done, showed hemobilia. CT abdomen was done on an emergency basis and showed active contrast extravasation from the cystic artery with demonstration of a pseudoaneurysm. The volume of the bleed was assessed using software (Fig. 2).

Total extravasated contrast volume including the pseudoaneurysm was estimated to be $7.7 \mathrm{cc}$, pseudoaneurysm measuring $2.6 \mathrm{cc}$ and extravasated contrast $5.1 \mathrm{cc}$. As the images have been obtained at an interval of 80 seconds, the rate of bleeding can be calculated to be $0.063 \mathrm{cc} / \mathrm{s}$ or $3.8 \mathrm{cc} / \mathrm{min}$. The patient underwent emergency laparotomy and ligation of bleeding cystic artery. Intra-operatively around $3500 \mathrm{ml}$ of blood with clots were noted in the gall bladder fossa. The patient gradually recovered from the hemorrhagic shock, following surgery.

\section{Case 3}

62-year-old male, had undergone endoscopic adrenaline infiltration for actively bleeding gastric ulcer. He presented again to the emergency department the next day with hematemesis. There was sudden drop in hemoglobin values from $6.7 \mathrm{~g} / \mathrm{dl}$ to $5.2 \mathrm{~g} / \mathrm{dl}$ over a span of 10 hours. Endoscopy showed active ooze from the base of the duodenal ulcer (Forrest classification Grade Ia). Emergency CT abdomen was undertaken which showed active bleed into the duo-
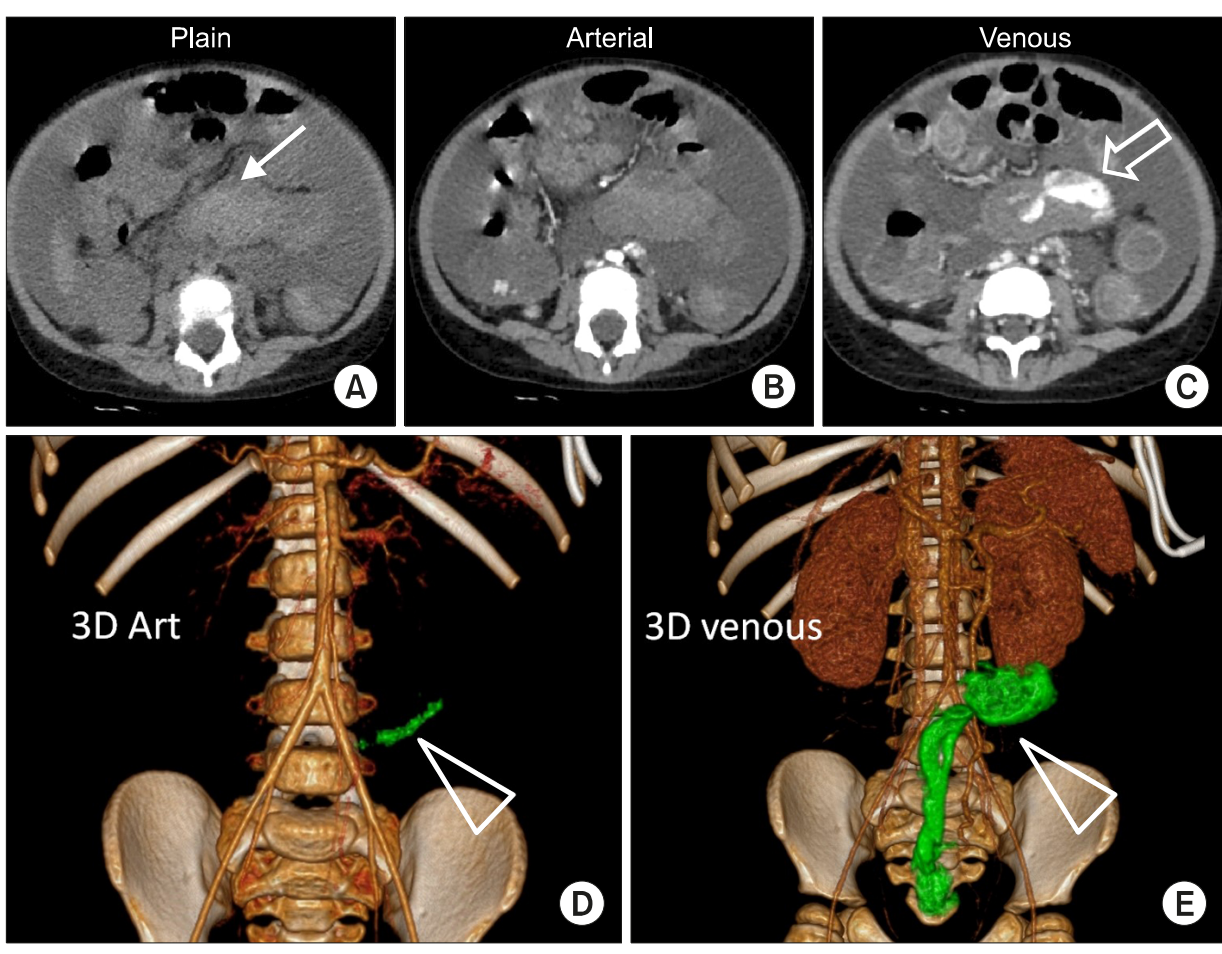

Fig. 1. (A) Plain CT image showing hyper-dense content in the sigmoid colon, suggestive of clots (arrow). (B) Arterial phase image shows no enlarged feeder artery. (C) Demonstrate extravasated contrast into the sigmoid lumen (open arrow). (D) Volume rendered image in the arterial phase show minimal contrast pooling in the sigmoid colon (triangle). Volume of extravasated contrast was estimated to be 0.3 cc. (E) Venous phase images of the same patient obtained 50 seconds later shows significant increase in pooled contrast within the sigmoid and rectal lumen (green, $15.5 \mathrm{cc}$ ). 

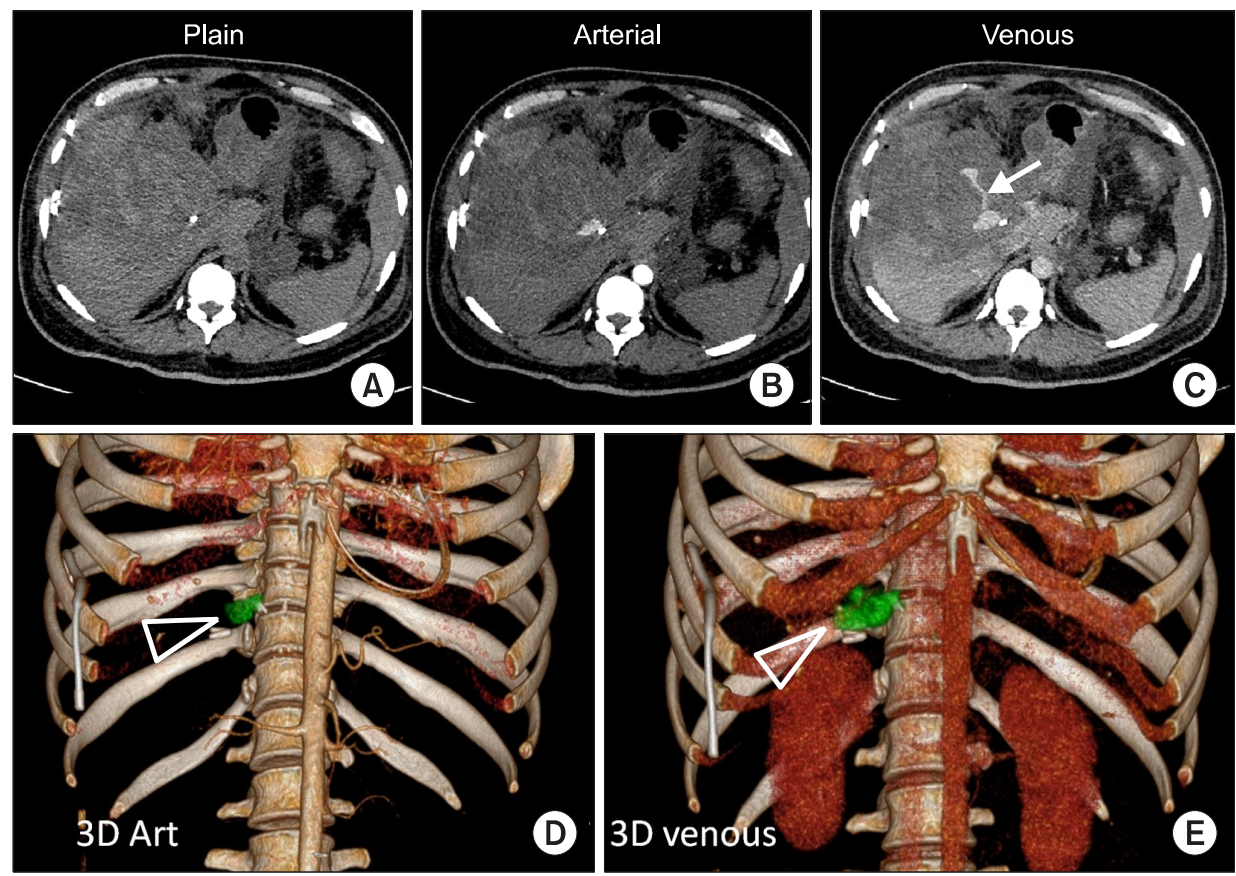

Fig. 2. (A) Plain CT image shows a metallic density (surgical clip) in the region of gall bladder fossa with a heterogeneous, hypodense perihepatic collection along right lateral surface of liver. (B) Arterial phase image shows a pseudo-aneurysm. (C) Demonstrates contrast extravasation in the venous phase images. (D) Volume rendered images of arterial phase acquisition show pseudoaneurysm of cystic artery (in green). Volume $2.6 \mathrm{cc}$. (E) Venous phase images of the same patient obtained 80 seconds later shows contrast pooling around the cystic artery along with the pseudoaneurym (triangle). The combined volume of pseudoaneurysm and extravasated contrast was calculated to be $7.7 \mathrm{cc}$. Left sided pigtail catheter can also be seen in the image.
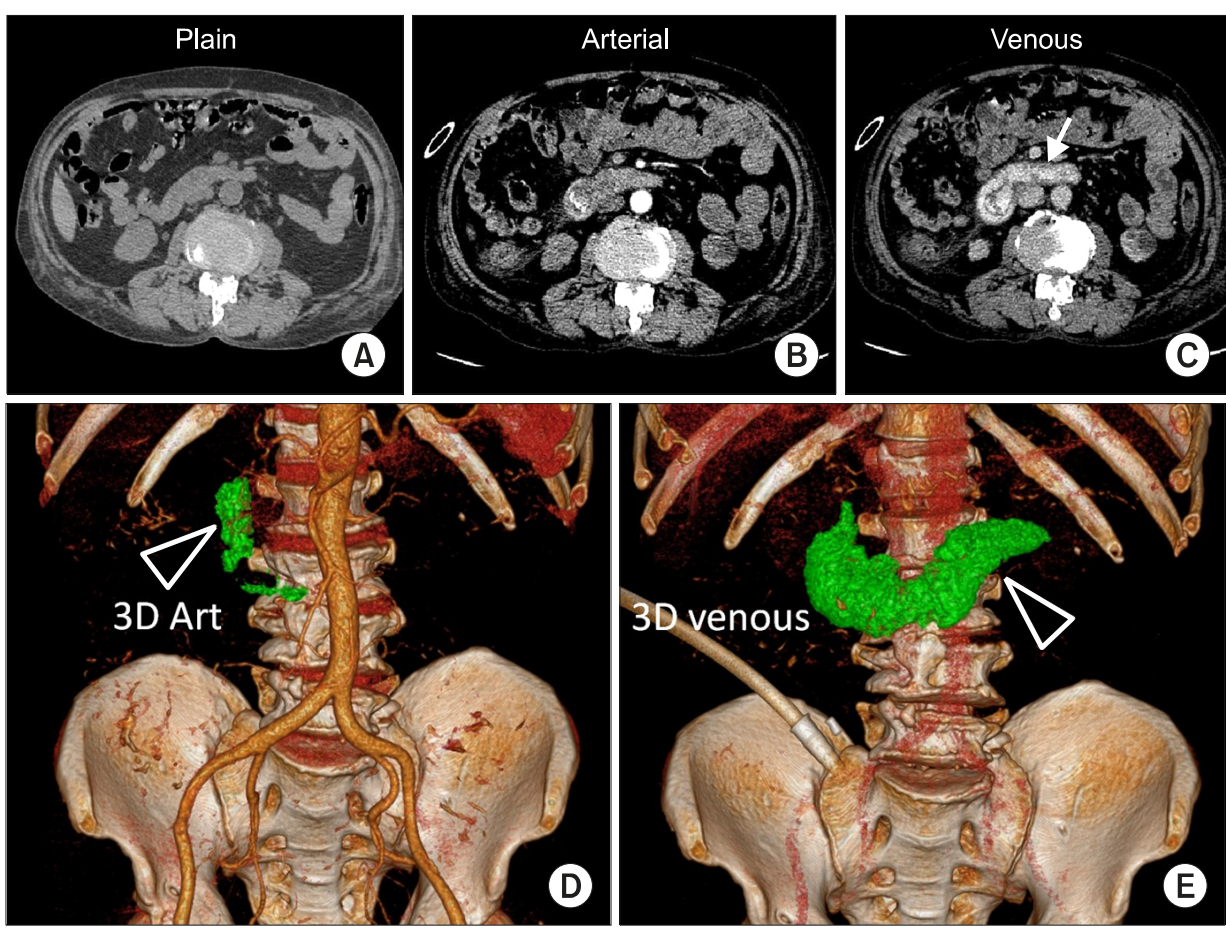

Fig. 3. (A) Plain CT image of upper abdomen appear normal. (B) Arterial phase image show contrast pooling within the third part of duodenum. (C) Delayed images obtained 70 seconds later showing significant pooling of contrast in the duodenum. (D) Volume rendered images of arterial phase acquisition showing mild contrast pooling in the second and third parts of duodenum (volume $4.8 \mathrm{cc}$ ). (E) Venous phase images obtained 70 seconds later shows significant increase in the quantity of pooled contrast (triangle). Volume $52.2 \mathrm{cc}$. denum (Fig. 3).

On volumetric estimation of extravasated contrast, between the two phases done 70 seconds apart was found to be $47.6 \mathrm{cc}$. Hence the rate of bleeding was calculated as $0.68 \mathrm{cc} / \mathrm{sec}$ (or $40.8 \mathrm{cc} / \mathrm{min}$ ). The patient underwent emergent angiography of abdomen which showed significant active 
bleeding from the gastroduodenal artery. Embolization of bleeding branch of gastroduodenal artery was then performed with large PVA particles (700-1000 microns). Patient was stabilised hemodynamically, showed clinical improvement.

MCECT examination provided estimation of amount and rate of bleed in all the above cases, thus guiding therapy. CT helped to execute a rapid definitive treatment which otherwise may have been delayed or lead to sub-optimal management. Prompt management was undertaken either by endoscopy, surgery or angiography as per the clinical decision. All patients showed clinical confirmation of bleeding. However, absolute confirmation of quantity of bleeding could not be performed by any of the performed methods.

\section{DISCUSSION}

MCECT is a very effective initial modality in the diagnosis of active gastrointestinal bleeding particularly in cases where endoscopy has failed to localize the site of bleed. The volumetric assessment of bleeding enhances the utility of CT, thus help in stratifying patients of differing severity of bleed. Such estimation of bleeding rate and volume has implications for the method of treatment and more importantly, the rapidity and urgency with which treatment is implemented.

Volumetric assessment of bleed or hematoma on abdominal imaging has not been explored adequately. CTbased method to quantify bleeding volume on plain CT has been explored in the study by Chikamatsu et al. ${ }^{8}$ in the context of post-biopsy renal bleeding. However we have not come across, on reviewing the literature, similar estimation of gastrointestinal bleed on MCECT. Detection of bleed on CT examination is based on the attenuation differences between bleeding focus and adjacent tissues. Haemoglobin has a higher Hounsfield Units (HU), hence can be detected if sufficient quantity is accumulated in organs where there is possibility for high intrinsic tissue contrast. Thus detection rate is highly variable depending upon the site, size, rate of bleed and suitable anatomy. Non-contrast CT estimation of bleed in GI bleed is ineffective due to small quantity and complex environment. Intravenous contrast can improve detection, (due to high HU of iodine) if detectable quantity is extravasated at bleeding site. Temporal changes in the quantity of detected bleed will help in estimating rate of bleeding. Improved reconstruction methods, computational techniques offered in the state of the art work stations facilitate predictable detection in a clinical setting. We have used Philips Intellispace WS, Ver 11 with facility for seeding an area of interest and region growing as defined by the set HU. Thus in our study we were able to predict the volume and rate of bleeding by setting the $\mathrm{HU}$ at $90+$ unit. We have noted that rate of bleed can be estimated in low rates $[3.8 \mathrm{cc} /$ $\min (0.063 \mathrm{cc} / \mathrm{sec})]$ as well as high rates $[40 \mathrm{cc} / \mathrm{min}(0.67$ $\mathrm{cc} / \mathrm{sec})]$. Temporal imaging with multiple phases is of value here to detect minimal amount of pooled contrast as an indicator of active haemorrhage. CT can visualize both intra-luminal and extra-luminal bleed. Estimated volume and rate of bleed provides significant additional insight in complex clinical scenarios. Estimated rate of bleed, along with clinical parameters provide good idea of the ongoing hemodynamic status.

The fact that no bowel preparation is needed for CT evaluation gives it a significant advantage over other modalities in evaluation of patients in acute state. The examination time required for $\mathrm{CT}$ is around 5 minutes with image-processing time of around 10-15 minutes. Thus the total examination time is usually around 15-20 minutes.

Clinical scoring systems for estimation of severity, risk of re-bleeding and mortality of upper gastrointestinal bleeding include Forrest classification, Rockall score, Baylor Bleeding score, Spanish Almela score and PNED score. These scoring methods comprise of components from patient history, clinical variables and endoscopic findings, with significant emphasis on endoscopic findings. ${ }^{9}$ Clinical risk scores are also used to identify high-risk patients requiring immediate intervention and low-risk patients who can be safely discharged. The major clinical scoring systems reported in literature are the clinical Rockall score and the Glasgow-Blatchford Score (GBS). Studies from several countries have suggested that the relatively simple GBS is superior to the Rockall score, at admission, in predicting clinically relevant end points. ${ }^{10}$

In clear contrast with upper GI bleeding, very few reports have focused on the prediction of outcomes in patients with lower GI bleeding. The existing scores like Oakland score, GBS, Rockall post endoscopy score, BLEED score, Strate score and NOBLADS score predict mortality, 
severity of bleeding and need for intervention. However data from these scoring systems are scarce and more studies are needed to establish its validity. ${ }^{11}$

\section{LIMITATIONS}

Our study has several limitations. CT detection methods are not very sensitive in detection of small bleed in anatomically complex regions. Low rates of bleeding may be overlooked and intermittent bleeding may not be detected by this method. This method also requires familiarity with volumetric assessment, though the learning curve is not steep. There are individual variations in rate of bleed and detection depending upon, $\mathrm{Hg}$ level, circulating blood volume, body surface area and general haemodynamic status of the patient during the examination. ${ }^{8}$ The CT estimation of rate of bleeding can be subjectively validated by endoscopy, surgery or angiography respectively as illustrated in our cases. However objective assessment of the same is not possible using the above methods. Since there is a need for urgent management, absolute estimation of bleeding rates using nuclear scintigraphy are also not useful.

Some discrepancy on the observations is expected in the context of bleed as it is progressive with a variable course. Time difference between CT and definitive therapy will alter clinical status. Our calculation is based on the assumption that rate of bleeding is constant, which need not be the case. Also our technique is more sensitive to bleed in the arterial phase, thus may underestimate slow venous (portal or systemic venous) bleeding. Another shortcoming of this technique is that metallic artifacts can potentially interfere with visualization of contrast in the bowel lumen on CT angiography and may lead to inaccurate assessment. Such limitations are well documented in CT angiography ${ }^{12}$ Further limitations, though less relevant in acute setting include dose, and risks affiliated with contrast material such as allergy, nephropathy, or hyperthyreosis. ${ }^{3}$ There is a scope for material imaging in quantification of GI bleed. ${ }^{4}$ It allows more accurate of measurement of macromolecules and can image iodine as a separate entity when measuring rate of bleeding during CT angiography. Material imaging is likely to enhance the sensitivity of quantitative volume measurement by more accurately measuring subtle, slow bleed in the venous phases.

The estimation of bleed volume on $\mathrm{CT}$ proves superior to clinical scoring systems as it can identify and stratify the rate of bleeding regardless of its aetiology, site and whether bleeding is intra or extra luminal.

\section{CONCLUSION}

Volumetric estimation of GI bleed on MCECT is a novel concept in the evaluation of gastrointestinal bleeding, with an immense potential. Methods identify the bleed site with prediction on rate of bleed, thus help stratifying patient for management. We hope that with routine use of material imaging and more user friendly software options for volume estimation, this technique will be a standard practice in emergency GI bleed management.

\section{ACKNOWLEDGEMENTS}

Authors would like to thank and acknowledge our colleagues from Gastroenterology and GI surgery team for the clinical input and academic support.

\section{ORCID}

Amritha P. P.: https://orcid.org/0000-0003-2598-7705

Karthik Gadabanahalli: https://orcid.org/0000-0001-5923-5902

Venkatraman Bhat: https://orcid.org/0000-0002-0220-2584

Kishore Kumar B. N.: https://orcid.org/0000-0001-5569-8926

\section{AUTHOR CONTRIBUTIONS}

Conceptualization: KG, VB. Data curation: APP, KKBN, VB. Formal analysis: APP, KG, VB, KKBN. Funding acquisition: N/A. Methodology: KG, KKBN. Project administration: KG, VB. Visualization: KG, VB. Writing - original draft: APP. Writing: APP, VB. Review \& amp; editing: VB, KKBN, KG.

\section{REFERENCES}

1. Zhao Y, Encinosa W. Hospitalizations for gastrointestinal bleeding in 1998 and 2006: statistical brief \#65. Rockville: Agency for Healthcare Research and Quality (US); 2008 [cited 2008 Dec]. Available from: http://www.ncbi.nlm.nih.gov/pubmed/21595135.

2. Gunjan D, Sharma V, Rana SS, Bhasin DK. Small bowel bleeding: a comprehensive review. Gastroenterol Rep (Oxf) 2014;2:262275.

3. Wu LM, Xu JR, Yin Y, Qu XH. Usefulness of CT angiography 
in diagnosing acute gastrointestinal bleeding: a meta-analysis. World J Gastroenterol 2010;16:3957-3963.

4. Wells ML, Hansel SL, Bruining DH, Fletcher JG, Froemming AT, Barlow JM, et al. CT for evaluation of acute gastrointestinal bleeding. Radiographics 2018;38:1089-1107.

5. Ren JZ, Zhang MF, Rong AM, Fang XJ, Zhang K, Huang GH, et al. Lower gastrointestinal bleeding: role of 64-row computed tomographic angiography in diagnosis and therapeutic planning. World J Gastroenterol 2015;21:4030-4037.

6. Artigas JM, Martí M, Soto JA, Esteban H, Pinilla I, Guillén E. Multidetector CT angiography for acute gastrointestinal bleeding: technique and findings. Radiographics 2013;33:1453-1470.

7. Kleinman JT, Hillis AE, Jordan LC. ABC/2: estimating intracerebral haemorrhage volume and total brain volume, and predicting outcome in children. Dev Med Child Neurol 2011;53: 281-284.
8. Chikamatsu Y, Matsuda K, Takeuchi Y, Kagaya S, Ojima Y, Fukami H, et al. Quantification of bleeding volume using computed tomography and clinical complications after percutaneous renal biopsy. Clin Kidney J 2017;10:9-15.

9. Monteiro S, Gonçalves TC, Magalhães J, Cotter J. Upper gastrointestinal bleeding risk scores: who, when and why? World $\mathrm{J}$ Gastrointest Pathophysiol 2016;7:86-96.

10. Stanley AJ. Update on risk scoring systems for patients with upper gastrointestinal haemorrhage. World J Gastroenterol 2012;18: 2739-2744.

11. Cañamares P, Alfaro E, Lanas A. Safe hospital discharge based on lower GI bleeding scores: a long way to go. AME Med J 2017;2:147.

12. Yoon W, Jeong YY, Kim JK. Acute gastrointestinal bleeding: contrast-enhanced MDCT. Abdom Imaging 2006;31:1-8. 